\title{
miR-34a targets the inhibin beta $B$ gene, promoting granulosa cell apoptosis in the porcine ovary
}

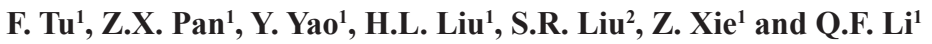

${ }^{1}$ College of Animal Science and Technology, Nanjing Agricultural University, Nanjing, China

${ }^{2}$ Animal Husbandry and Veterinary Institute,

Xinjiang Academy of Agricultural and Reclamation Science, Shihezi, China

Corresponding authors: Z. Xie / Q.F. Li

E-mail: zxie@njau.edu.cn / liqifa@njau.edu.cn

Genet. Mol. Res. 13 (2): 2504-2512 (2014)

Received June 17, 2013

Accepted October 10, 2013

Published January 14, 2014

DOI http://dx.doi.org/10.4238/2014.January.14.6

\begin{abstract}
During ovarian follicular growth and development, only a few follicles actually ovulate. Recently, it was found that follicular atresia is triggered by granulosa cell apoptosis, but the molecular mechanism of follicular atresia was not understood. Using flow cytometry, we found that miR-34a promotes granulosa cell apoptosis in pig ovarian follicles. In addition, inhibin beta B was found to be a miR34a target gene, based on luciferase reporter assays, quantitative RTPCR and Western blotting. Taken together, our data indicate that miR34a plays an important role in granulosa cell apoptosis by targeting the INHBB gene in the porcine ovary.
\end{abstract}

Key words: miR-34a; INHBB; Apoptosis; Granulosa cells 


\section{INTRODUCTION}

Follicles are the functional units of the ovary, and each follicle consists of an oocyte surrounded by one or more layers of somatic granulosa cells. Several hundred thousand primordial follicles are present in the mammalian ovary, but only a limited number develop to the pre-ovulatory stage, and then finally ovulate. During follicular growth and development, more than $99 \%$ of follicles undergo degeneration known as "follicular atresia" at any stage of growth and development (Tilly et al., 1991; Maeda et al., 2007). A recent study indicated that follicular atresia is triggered by granulosa cell apoptosis (Manabe et al., 2008), but the actual molecular mechanism of follicular atresia is still unknown.

miRNAs are a class of small non-coding 18- to 25-nucleotide-long RNAs that negatively regulate gene expression by binding to the 3'-untranslated region (3'-UTR) of target messenger RNAs; this causes translational repression or degradation (ValenciaSanchez et al., 2006; Cochrane et al., 2012). Information on the roles of miRNAs in controlling gonadal functions is limited to characterization of the expression profiles of miRNAs in normal ovarian cells (Ro et al., 2007) and in ovarian carcinoma cells (Iorio et al., 2005; Corney et al., 2007). Ro et al. (2007) profiled the mouse ovarian miRNAs and concluded that these miRNAs may play critical roles in controlling the expression of genes essential for ovarian folliculogenesis and endocrine function. These studies suggest that miRNA may play an important role in follicular development, but the mechanism of miRNA regulation remains to be elucidated.

Recently, miRNAs have been identified and thoroughly characterized in mouse oocytes and early embryos by several independent groups (Tang et al., 2007; Murchison et al., 2007). Interestingly, specific deletion of dicer1, the key ribonuclease that cleaves pre-miRNAs to mature length miRNAs, results in depletion of many of the miRNAs identified in the mouse oocyte (Watanabe et al., 2006). These mutant oocytes also fail to complete maturation resulting in a block at first cleavage following fertilization (Miles et al., 2012).

In our previous study, we found that miR-34a was upregulated during follicular atresia using a miRNA microarray assay in porcine ovarian follicles. In this study, we found that miR-34a could induce pig granulosa cell apoptosis in vitro, and that inhibin beta $\mathrm{B}$ (INHBB) is a direct target gene of miR-34a.

\section{MATERIAL AND METHODS}

\section{Cell culture}

HeLa cells were cultured at $37^{\circ} \mathrm{C}$ in a humidified $5 \% \mathrm{CO}_{2}$ atmosphere in Dulbecco's modified Eagle's medium (DMEM) (Gibco, Shanghai, China) supplemented with 10\% (v/v) fetal bovine serum (FBS, Gibco), $100 \mathrm{U} / \mathrm{mL}$ penicillin, and $100 \mu \mathrm{g} / \mathrm{mL}$ streptomycin (Sigma, Shanghai, China). Granulosa cells were collected from porcine ovaries using a syringe; they were washed with PBS and cultured in DMEM/F12 (1:1) with 15\% fetal bovine serum in an incubator with $5 \% \mathrm{CO}_{2}$ in air at $37^{\circ} \mathrm{C}$. Penicillin $(100 \mathrm{U} / \mathrm{mL})$ and streptomycin $(100 \mu \mathrm{g} / \mathrm{mL})$ were used in the cultures. 


\section{Transfection}

miR-34a mimics, miR-34a negative control (miR-NC) and miR-34a inhibitor (InmiR-34a) were synthesized and purified by GenePharma Co. (Shanghai, China). Transfection was performed with Lipofectamine 2000 Reagent (Invitrogen, Shanghai, China), following manufacturer protocol. Briefly, cells were seeded in 12- or 6- well plates one day prior to transfection. When the cells reached 50-70\% confluence, miRNAs were transfected into the cells at different final concentrations. All experiments were performed in triplicate.

\section{Vector construction and dual-luciferase reporter assay}

The 3'-UTRs of INHBB containing miR-34a binding sites were cloned downstream of the luciferase reporter in the pmirGLO Dual-Luciferase miRNA Target Expression Vector (Promega, Madison, USA). HeLa cells were plated in 12-well plates and transfected with $20 \mathrm{nM}$ miRNA mimics and with $1000 \mathrm{ng}$ luciferase vector (pmirGLO). The cells were harvested at $24 \mathrm{~h}$ post-transfection, and the luciferase activity was measured using the Dual-Glo luciferase assay kit (Promega, Madison, WI, USA).

\section{Real-time PCR}

Total RNA was extracted using TRIZOL, and the RT reactions were performed with MLV, according to manufacturer protocol. Real-time PCR was performed with SYBR Premix Ex Taq in a reaction volume of $20 \mu \mathrm{L}$. Primer sequences were as follows: INHBB, forward: 5'-CGTGGTGCCCGTGTTTGT-3' and reverse: 5'-TGCCCTCACAGTAGTTCCCATA-3', 227bp; GAPDH, forward: 5'-GGACTCATGACCACGGTCCAT-3' and reverse: 5'-TCAGATCCACAACCGACACGT-3', 220 bp. GAPDH was used as normalization control, and the relative expression levels were calculated using the $2^{-\Delta \Delta \mathrm{Ct}}$ method (Livak and Schmittgen, 2001).

\section{Apoptosis analysis}

Granulosa cells were transfected with the miR-34a mimics or miR-NC as a control. After an additional $48 \mathrm{~h}$ incubation, the cells were harvested, then washed twice with pre-chilled PBS and resuspended in $100 \mu \mathrm{L} 1 \mathrm{X}$ binding buffer at a concentration of $1 \mathrm{x}$ $10^{5}$ cells $/ \mathrm{mL}$. Annexin $\mathrm{V}$ and propidium iodide (PI) double-staining was performed using the Annexin V-FITC Apoptosis Detection kit (BD Biosciences, San Jose, CA, USA) according to manufacturer protocol. The apoptosis analysis was performed in the BD LSRII Flow Cytometer System with FACS Diva Software within $1 \mathrm{~h}$.

\section{Western blotting}

Cell lysate was subjected to SDS-PAGE and transferred to a nitrocellulose membrane. Protein expression was analyzed by Western blotting with primary antibodies against INHBB (sc-6307; Santa Cruz Biotechnology, Santa Cruz, CA, USA) and then 
incubated with a secondary antibody. Blots were finally developed with SuperSignal West Pico chemiluminescent substrate for $5 \mathrm{~min}$. The substrate was prepared by mixing the Super-Signal West Pico Stable Peroxidase Solution and the Super-Signal West Pico Luminol/Enhancer Solution at a ratio of 1:1. After draining the solutions, chemiluminescence was detected using a luminescent image analyzer LAS-4000 (Fujifilm, Tokyo, Japan).

\section{Statistical analysis}

All results are reported as means $\pm \mathrm{SE}$. The statistical analysis was performed with a $t$-test to compare two groups, and an analysis of variance (ANOVA) was applied for multiple comparisons. Differences were considered to be significant at $\mathrm{P}<0.05$. All data in the study were evaluated using SPSS 18 (SPSS Inc., USA).

\section{RESULTS}

\section{INHBB is a target of miR-34a}

INHBB, one of the predicted miR-34a targets, contains miR-34a binding sites in INHBB 3'-UTR (Figure 1A). To further investigate the cellular effect of miR-34a on its potential targets INHBB, we hypothesized that miR-34a represses INHBB expression through this site, so we constructed one reporter vector including luciferase cDNA followed by an miR-34a binding site (Figure 1B and C). The reporter vector was co-transfected into HeLa cells with miR-34a mimics, In-miR-34a or miR-NC as a control. miR-34a decreased luciferase activity of the reporter vector, but not the reporter with miR-NC. In contrast, In-miR-34a upregulated luciferase activity (Figure 1D). Taken together, miR-34a inhibited INHBB expression directly by binding to its mRNA.

\section{miR-34a promotes granulosa cell apoptosis in vitro}

Porcine granulosa cells were transfected with the miR-34a mimics, or miR-NC. After a 48-h incubation, we determined the effect of miR-34a on cultured granulosa cell in vitro. Anti-annexin-V PI staining and FACS analysis confirmed that cell apoptosis significantly increased after transfection of the miR-34a mimics (Figure 2A and B). These data suggested that miR-34a promoted granulosa cell apoptosis.

\section{miR-34a downregulated INHBB expression in granulosa cells}

To determine whether INHBB gene is a true target of miR-34a, granulosa cells were transfected with miR-34a mimics, In-miR-34a or miR-NC. Immunoblotting and real-time PCR analysis revealed that INHBB protein (Figure 3A) but not INHBB mRNA (Figure 3B) expression was significantly lower in miR-34a transfected granulosa cells compared with the control cells, whereas the In-miR-34a-treated cells were able to increase INHBB protein expression (Figure $3 \mathrm{C}$ ). These results confirmed that INHBB gene was a miR-34a target. miR-34a regulated INHBB gene expression at the posttranscriptional level (Figure 3B). 
A

Human INHBB 3' UTR

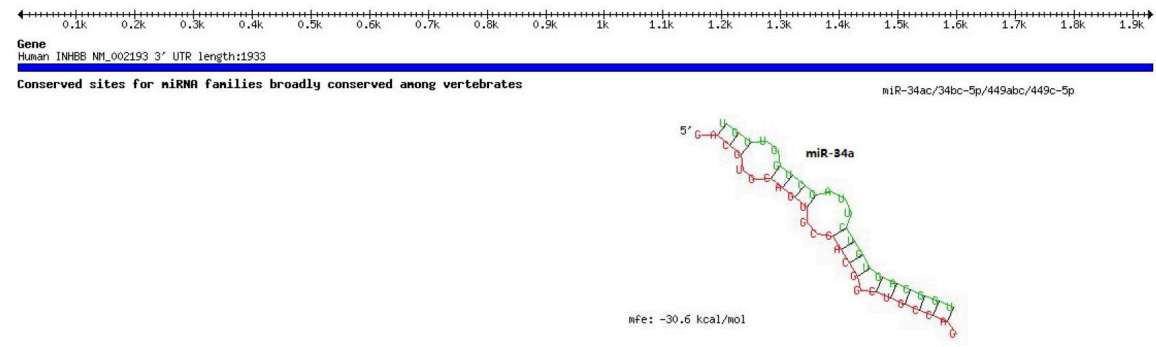

B

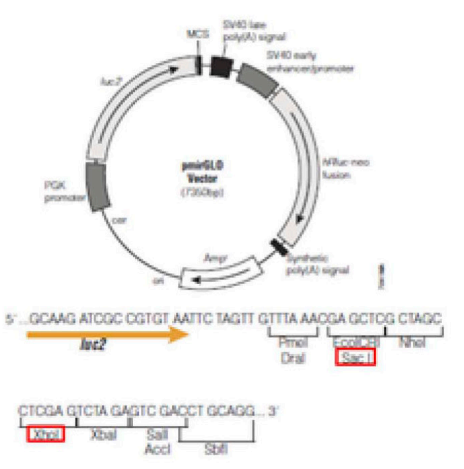

C

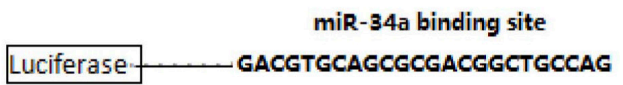

D

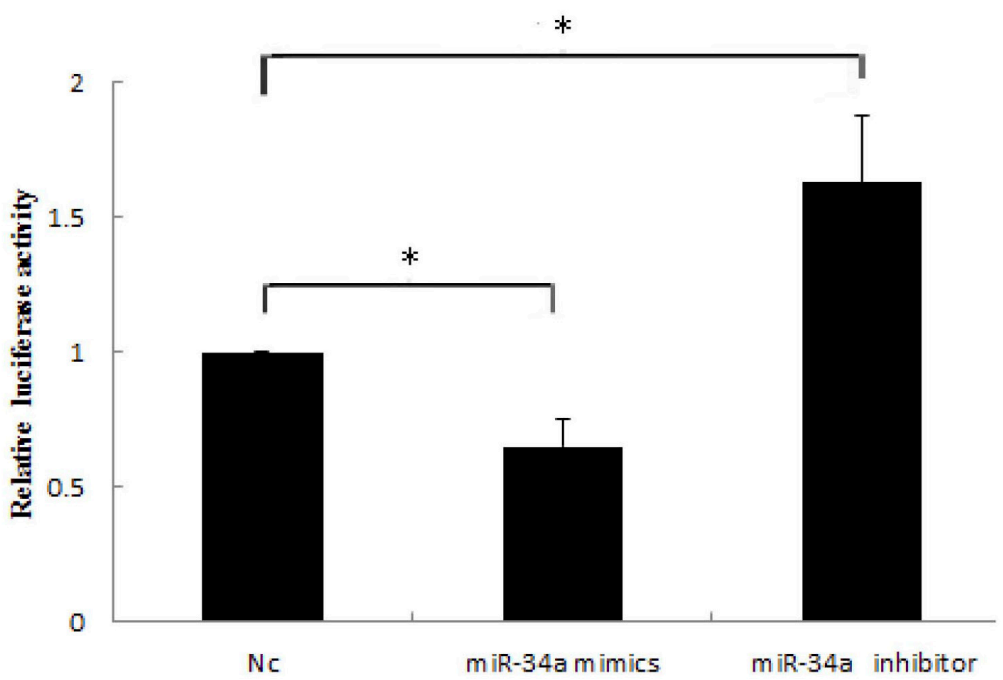

Figure 1. miR-34a downregulated INHBB expression in HeLa cells. A. Diagram of the INHBB 3'-UTR with potential binding-sites of miR-34a and a miR-34a binding site within INHBB mRNA was predicted by RNA hybrid. B. Map of the pmirGLO luciferase reporter vector. The red rectangles show the restrictive endonuclease used for cloning. C. miR-34a binding sequence for INHBB gene. D. Luciferase activity assay. HeLa cells were transfected with luciferase vectors and the miR-34a mimics, In-miR-34a or. miR-NC and harvested for the dual luciferase reporter assay after $24 \mathrm{~h} .\left({ }^{*} \mathrm{P}<0.05\right.$, mean significant difference following a two-tailed Student's t-test). 
A

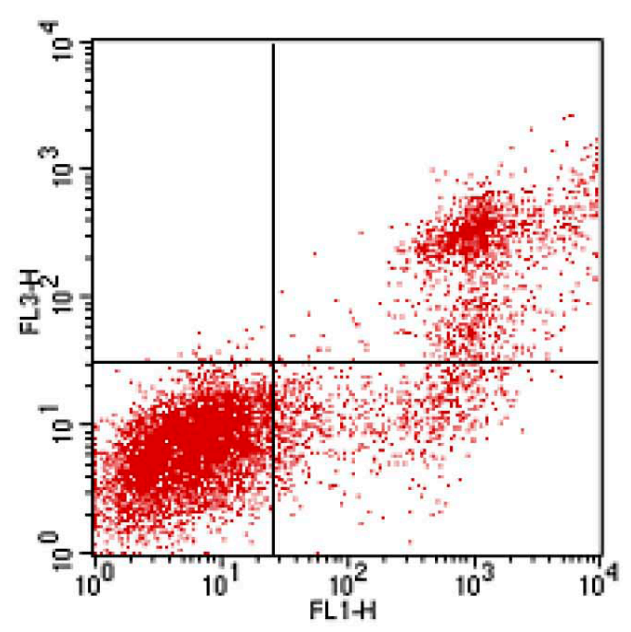

miR-34a

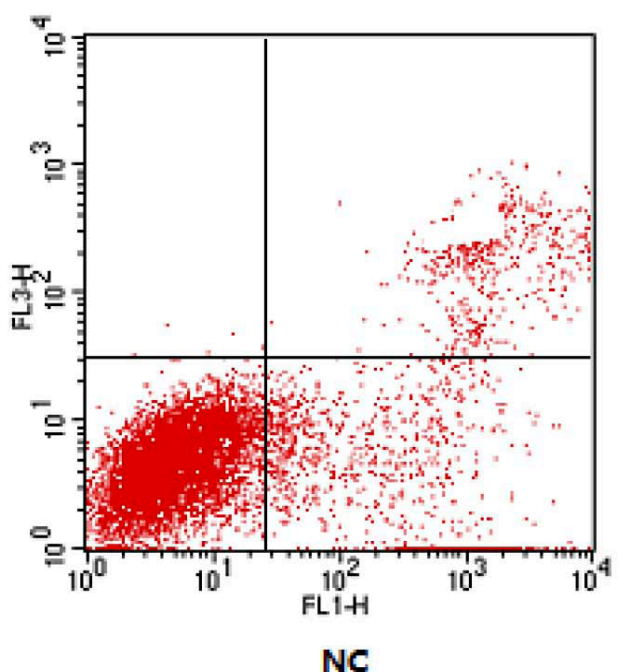

NC

B

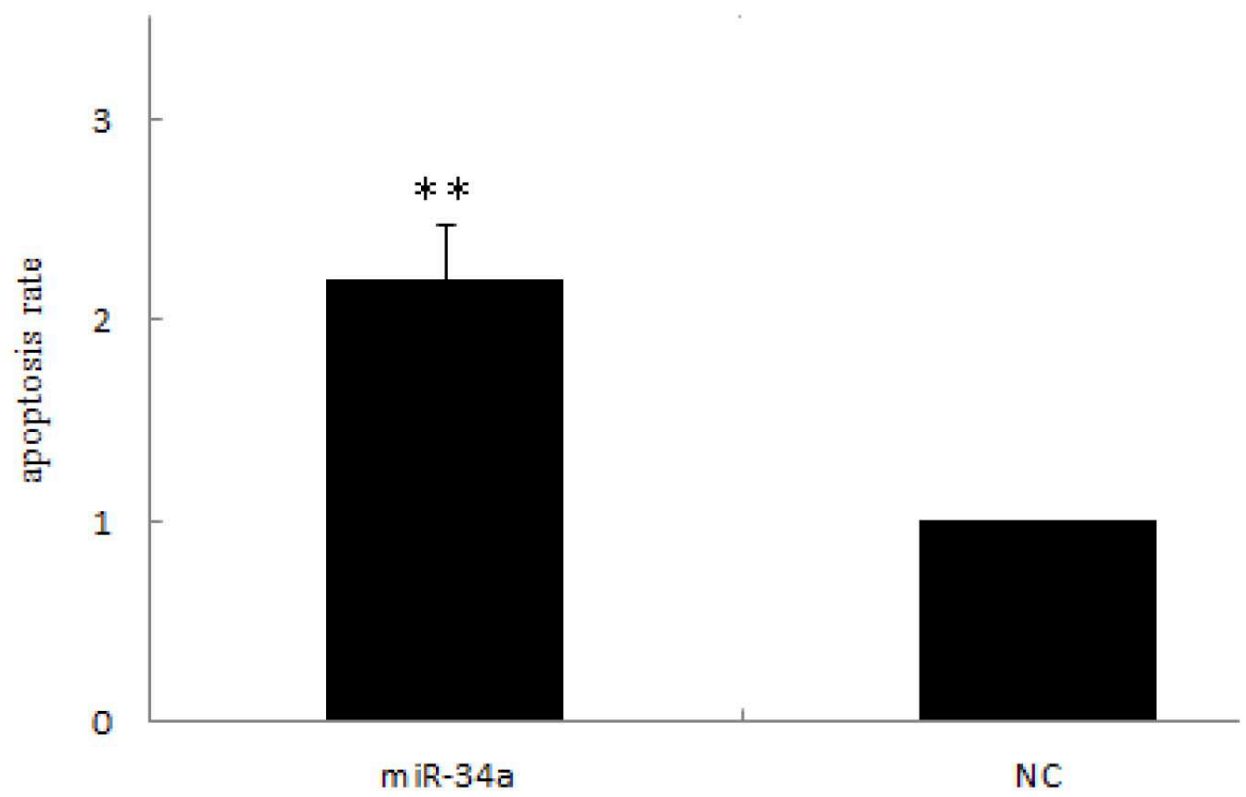

Figure 2. miR-34a promotes granulosa cells apoptosis in vitro. A. B. Flow cytometry analysis (FACS) of apoptosis. After treatment and additional culture, cells were harvested and stained with Anti-annexin V-propidium iodide followed by FACS analysis. Apoptosis improved significantly following the miR-34a transfection. (**P $<0.01$ mean great significant difference following a two-tailed Student's t-test). 


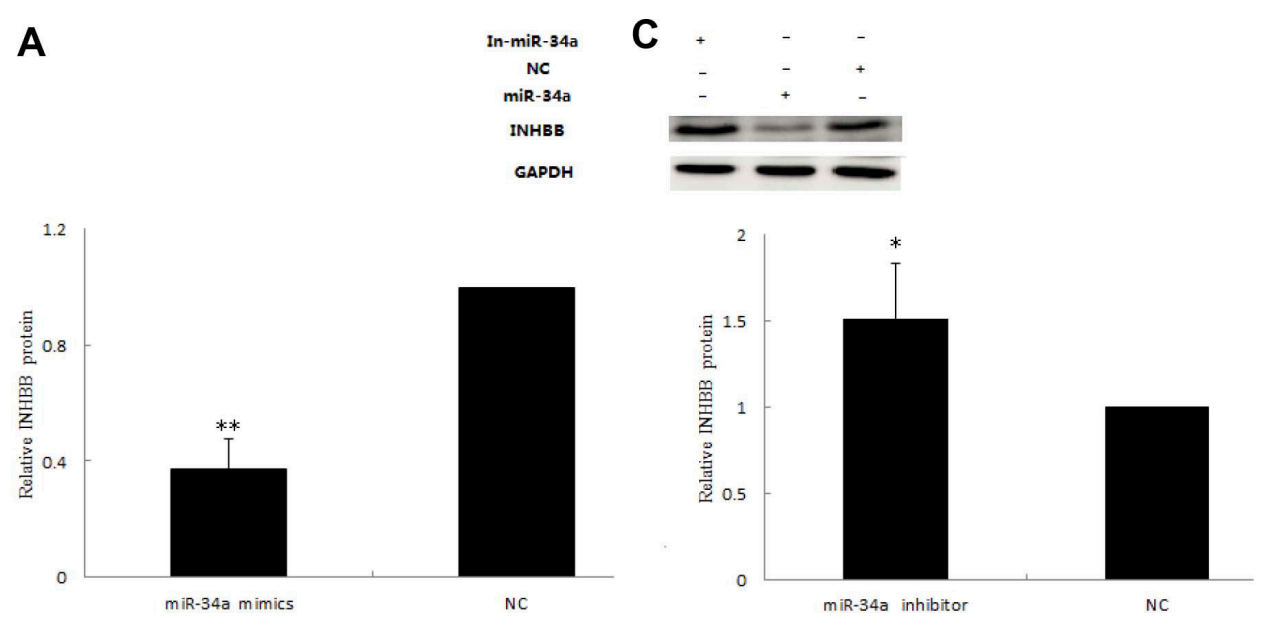

\section{B}

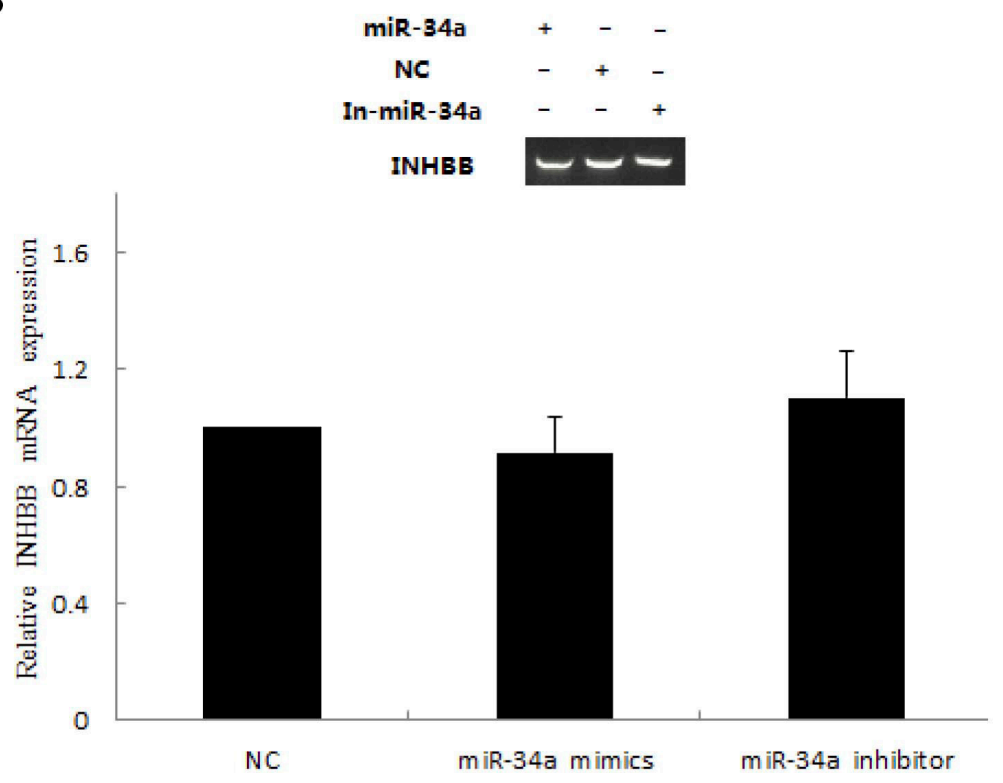

Figure 3. INHBB is a target of miR-34a in granulosa cells. A. C. Relative INHBB protein expression in granulosa cells transfected with miR-34a mimics, In-miR-34a or miR-NC. B. Relative INHBB mRNA expression in granulosa cells transfected with miR-34a mimics, In-miR-34a or miR-NC. ( ${ }^{*} \mathrm{P}<0.05, * * \mathrm{P}<0.01$ separately mean significant and great significant difference following a two-tailed Student's t-test).

\section{DISCUSSION}

MiRNA regulation of gene expression plays a role in development, differentiation, proliferation, and apoptosis. For example, miR-34a inhibition of silent information regulator 
one could regulate cell apoptosis (Yamakuchi et al., 2008). When miR-34a was transfected into porcine granulosa cells, cellular apoptosis increased, indicating that miR-34a could regulate cell growth by interfering with the expression of proteins linked to cell proliferation or apoptosis.

MiR-34s form an evolutionarily conserved miRNA family, with three members in vertebrate genomes (miR-34a, miR-34b, and miR-34c) and single orthologues in invertebrate species. It is known that ectopic expression or delivery of synthetic miR-34 results in cell cycle arrest or senescence (Bommer et al., 2007; Hermeking, 2007; Tazawa et al., 2007). MiR-34 induces growth arrest through its activation of the cyclin-dependant kinase inhibitor p21. Notably, it has been shown that miR-34 can induce G1 arrest (Bommer et al., 2007; Hermeking, 2007). MiR-34s are known to control broad programs of targets involved in cell cycle control, apoptosis, and DNA repair, among which cyclin-dependent kinase (cdk) 4, cyclin E2, cMet, cdk6, and bcl-2 have been identified as possible candidates (Hermeking, 2007; Tazawa et al., 2007). Recently, several direct targets of miR-34a have been found. Ji et al. (2008) showed that miR-34 directly targeted p53 and functioned as a tumor suppressor. MiR-34 is also known to target Notch, HMGA2, and Bcl-2, genes involved in the self-renewal and survival of cancer stem cells. Our results demonstrated that miR-34a promotes porcine granulosa cell apoptosis by targeting INHBB.

In summary, we found that the INHBB gene is a miR-34a target and that miR-34a regulates INHBB gene expression at the posttranscriptional level. These findings demonstrate that miR-34a promotes porcine granulosa cell apoptosis by targeting the INHBB gene in vitro.

\section{ACKNOWLEDGMENTS}

Research supported by the Fundamental Research Funds for the Central Universities (\#KYZ201152), the National Key Scientific Program (\#2007CB947403), and the National Natural Science Foundation of China (\#30901027).

\section{REFERENCES}

Bommer GT, Gerin I, Feng Y, Kaczorowski AJ, et al. (2007). p53-mediated activation of miRNA34 candidate tumorsuppressor genes. Curr. Biol. 17: 1298-1307.

Cochrane DR, Spoelstra NS and Richer JK (2012). The role of miRNAs in progesterone action. Mol. Cell Endocrinol. 357: 50-59.

Corney DC, Flesken-Nikitin A, Godwin AK, Wang W, et al. (2007). MicroRNA-34b and MicroRNA-34c are targets of p53 and cooperate in control of cell proliferation and adhesion-independent growth. Cancer Res. 67: 8433-8438.

Hermeking H (2007). p53 enters the microRNA world. Cancer Cell 12: 414-418.

Iorio MV, Ferracin M, Liu CG, Veronese A, et al. (2005). MicroRNA gene expression deregulation in human breast cancer. Cancer Res. 65: 7065-7070.

Ji Q, Hao X, Meng Y, Zhang M, et al. (2008). Restoration of tumor suppressor miR-34 inhibits human p53-mutant gastric cancer tumorspheres. BMC Cancer 8: 266.

Livak KJ and Schmittgen TD (2001). Analysis of relative gene expression data using real-time quantitative PCR and the 2[-Delta Delta C(T)] method. Methods 25: 402-408.

Maeda A, Inoue N, Matsuda-Minehata F, Goto Y, et al. (2007). The role of interleukin-6 in the regulation of granulosa cell apoptosis during follicular atresia in pig ovaries. J. Reprod. Dev. 53: 481-490.

Manabe N, Matsuda-Minehata F, Goto Y, Maeda A, et al. (2008). Role of cell death ligand and receptor system on regulation of follicular atresia in pig ovaries. Reprod. Domest. Anim. 43 (Suppl 2): 268-272.

Miles JR, McDaneld TG, Wiedmann RT, Cushman RA, et al. (2012). MicroRNA expression profile in bovine cumulusoocyte complexes: possible role of let-7 and miR-106a in the development of bovine oocytes. Anim. Reprod. Sci. 130: $16-26$. 
Murchison EP, Stein P, Xuan Z, Pan H, et al. (2007). Critical roles for Dicer in the female germline. Genes Dev. 21: 682693.

Ro S, Song R, Park C, Zheng H, et al. (2007). Cloning and expression profiling of small RNAs expressed in the mouse ovary. RNA 13: 2366-2380.

Tang F, Kaneda M, O'Carroll D, Hajkova P, et al. (2007). Maternal microRNAs are essential for mouse zygotic development. Genes Dev. 21: 644-648.

Tazawa H, Tsuchiya N, Izumiya M and Nakagama H (2007). Tumor-suppressive miR-34a induces senescence-like growth arrest through modulation of the E2F pathway in human colon cancer cells. Proc. Natl. Acad. Sci. U. S. A. 104: $15472-15477$.

Tilly JL, Kowalski KI, Johnson AL and Hsueh AJ (1991). Involvement of apoptosis in ovarian follicular atresia and postovulatory regression. Endocrinology 129: 2799-2801.

Valencia-Sanchez MA, Liu J, Hannon GJ and Parker R (2006). Control of translation and mRNA degradation by miRNAs and siRNAs. Genes Dev. 20: 515-524.

Watanabe T, Takeda A, Tsukiyama T, Mise K, et al. (2006). Identification and characterization of two novel classes of small RNAs in the mouse germline: retrotransposon-derived siRNAs in oocytes and germline small RNAs in testes. Genes Dev. 20: 1732-1743.

Yamakuchi M, Ferlito M and Lowenstein CJ (2008). miR-34a repression of SIRT1 regulates apoptosis. Proc. Natl. Acad. Sci. U. S. A. 105: 13421-13426. 